Symposium on Strongly Correlated Electron Systems, Int. Conf. on Magnetism, Cairns, Australia, Jul. 27-Aug. 1, 1997, JMMM

\title{
Magnetic correlations in a classic Mott system
}

Wei Bao, ${ }^{a}$ C. Broholm,${ }^{b}$ G. Aeppli, ${ }^{c}$ S. A. Carter,${ }^{d}$ P. Dai,${ }^{e}$ C. D. Frost,${ }^{f}$ J. M. Honig ${ }^{g}$ and P. Metcalf ${ }^{g}$

a Brookhaven National Laboratory, Upton, NY 11973, USA, ${ }^{b}$ The Johns Hopkins University, Baltimore, MD 21218, USA, ${ }^{c}$ NEC, 4 Independence Way, Princeton, NJ 08540, USA, ${ }^{d}$ University of California, Santa Cruz, CA 95064, USA, ${ }^{e}$ Oak Ridge National Laboratory, Oak Ridge, TN 37831, USA, ${ }^{f}$ ISIS, Rutherford Appleton Laboratory, Oxon, OX11 OQX, UK, ${ }^{g}$ Purdue University, West Lafayette, IN 47907, USA

\begin{abstract}
The metal-insulator transition in $\mathrm{V}_{2} \mathrm{O}_{3}$ causes a fundamental change in its magnetism. While the antiferromagnetic insulator (AFI) is a Heisenberg lo-

calized spin system, the antiferromagnetism in the strongly correlated metal is determined by a Fermi surface instability. Paramagnetic fluctuations in the metal and insulator represent similar spatial spin correlations, but are unrelated to the long range order in the AFI. The phase transition to the AFI induces an abrupt switching of magnetic correlations to a different magnetic wave vector. The AFI transition, therefore, is not a conventional spin orderdisorder transition. Instead it is accounted for by an ordering in the occupation of the two degenerate $d$-orbitals at the Fermi level.
\end{abstract} PECHOED

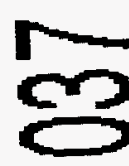

MAY O $\$ 1998$

Keywords: Mott localization, spin density wave, itinerant electrons - antiferromagnetism, orbital ordering, paramagnetic fluctuations

DTIC QDALTTY INEPECTED 1

"The submitted manuscript has been authored by a contractor of the U.S. Government under contract No. DE-AC05-96OR22464. Accordingly, the U.S. Government retains a nonexclusive, royalty-free license to publish or reproduce the published form of this contribution, or allow others to do so, for U.S. 
Some of the most fascinating members of strongly correlated electron systems are transition metal oxides. For example, superconductivity occurs at a temperature as high as $133 \mathrm{~K}$ at ambient pressure in $\mathrm{HgBa}_{2} \mathrm{Ca}_{2} \mathrm{Cu}_{3} \mathrm{O}_{8+\delta}$ [1]. Colossal magnetoresistance, where the resistivity is reduced by 2 orders of magnitude in a magnetic field of 6 Tesla, is realized in $(\mathrm{La}, \mathrm{Y}) \mathrm{MnO}_{3}$ [2]. In $\mathrm{V}_{2} \mathrm{O}_{3}$, the resistivity increases by 8 orders of magnitude at a metal-insulator transition [3]. At this stage, our theoretical knowledge of strongly correlated systems is rather sketchy and fragmental. Accumulating empirical knowledge with experiments is indispensable. Since static or dynamic magnetic correlations are natural consequences of strong Coulomb correlations and depend sensitively on electron wave functions, their direct measurement by neutron scattering can provide fresh insight about the electronic processes. In this paper, such an endeavor for the classic Mott system, pure and doped $\mathrm{V}_{2} \mathrm{O}_{3}[4]$, is reviewed.

A composition-temperature phase diagram for $\mathrm{V}_{2} \mathrm{O}_{3}$ is presented in Fig. 1. The metalinsulator transition in the paramagnetic phase is a classic example of the Mott-Hubbard transition [5]. Metallic $\mathrm{V}_{2} \mathrm{O}_{3}$ is a strongly correlated Fermi liquid with a Sommerfeld specific heat constant $\gamma=54-70 \mathrm{~mJ} / \mathrm{K}^{2}$ per mole $\mathrm{V}[6]$ and with a $\mathrm{T}^{2}$ dependence of resistivity below $\sim 40 \mathrm{~K}$ with a coefficient $0.03-0.05 \mu \Omega \mathrm{cm} / \mathrm{K}^{2}[7]$. There is also long range antiferromagnetic order in the doping-induced metal [8].

The magnetic structure in the antiferromagnetic metal was only recently determined by us using neutron diffraction [9]. It is a transverse incommensurate spin density wave (SDW) with a staggered moment of $0.15 \mu_{B}$ per vanadium (see Fig. 2). This small moment antiferromagnetic long range order is accompanied by dynamic magnetic correlations with a bandwidth more than 20 times $k_{B} T_{N}$ and an amplitude of more than $0.32 \mu_{B}$ per vanadium, substantially larger than the ordered moment. Short range magnetic correlations exist even at temperatures more than 20 times of the Néel temperature [10]. These results can not be explained by localized spin models. Instead they are analogous to the SDW in Cr metal [11]. However, in contrast to $\mathrm{Cr}$, only a small area of the Fermi surface is involved in the SDW in metallic $\mathrm{V}_{2-y} \mathrm{O}_{3}[9]$, and strong electron correlations in metallic $\mathrm{V}_{2} \mathrm{O}_{3}$ require only 


\section{DISCLAIMER}

This report was prepared as an account of work sponsored by an agency of the United States Government. Neither the United States Government nor any agency thereof, nor any of their employees, makes any warranty, express or implied, or assumes any legal liability or responsibility for the accuracy, completeness, or usefulness of any information, apparatus, product, or process disclosed, or represents that its use would not infringe privately owned rights. Reference herein to any specific commercial product, process, or service by trade name, trademark, manufacturer, or otherwise does not necessarily constitute or imply its endorsement, recommendation, or favoring by the United States Government or any agency thereof. The views and opinions of authors expressed herein do not necessarily state or reflect those of the United States Government or any agency thereof. 
near nesting of the Fermi surface for the SDW to occur.

Because of strong correlations, the Fermi velocity for metallic $\mathrm{V}_{2} \mathrm{O}_{3}$ is smaller than that for $\mathrm{Cr}$ metal by one order of magnitude [9]. This removes the problems in resolving the excitation spectrum which has plagued neutron scattering studies of $\mathrm{Cr}$. We have established unambiguously that the intrinsic dynamic spin correlation function, $S(\mathbf{q}, \omega)$, even at $1.4 \mathrm{~K}$ in the ordered AFM phase, consists of a single broad lobe at each antiferromagnetic Bragg point in the energy range, $1.5 \mathrm{meV} \leq \hbar \omega \leq 25 \mathrm{meV}$, which we have probed $[9,12]$. (see Fig. 3 ). These broad lobes represent spin excitations inside the Stoner electron-hole continuum. Their dominance in spin dynamics reflects the fact that metallic $\mathrm{V}_{2} \mathrm{O}_{3}$ is in the small moment limit, in other words, it is near the quantum critical point for itinerant antiferromagnetism $[10,13]$.

We have measured spin excitations throughout the Brillouin zone from $1.4 \mathrm{~K}$ to $200 \mathrm{~K}$ for a metallic sample, $\mathrm{V}_{1.973} \mathrm{O}_{3}\left(T_{N}=9 \mathrm{~K}\right)[10]$. By normalizing to neutron scattering from transverse phonons, the dynamic spin correlation function, $S(\mathbf{q}, \omega)$, was obtained in absolute units. We found that the $q, \omega$ and $T$ dependence of spin fluctuations can be described by the self-consistent renormalization (SCR) theory for itinerant antiferromagnetism in the small moment limit [14]. Thermodynamic properties below $\sim 10 T_{N}$ are quantitatively accounted for by this theory in its simplest form with four experimentally determined parameters [10].

Accompanied by a strongly first order antiferromagnetic transition as well as a structural transition, the metal-insulator transition from the paramagnetic metal (PM) to the AFI is considerably more complex than the metal-insulator transition in the paramagnetic phase. The mechanism for this phase transition has been controversial. We examined the PMAFI transition by investigating the spin dynamics of a pure $\mathrm{V}_{2} \mathrm{O}_{3}$ sample. The magnetic structure in the AFI [15] is composed of vertical ferromagnetic planes whose spin direction alternates with a wave vector in the basal plane (see Fig. 2). Magnetic excitations are conventional spin waves $[16,12]$ and magnetism inside the AFI phase can be accounted for by a conventional Heisenberg localized spin model. The antiferromagnetic transition, however, is not conventional. In the upper panel of Fig. 4, the two counter-propagating spin 
wave modes are shown by solid circles at $140 \mathrm{~K}$ in the AFI phase. When the sample is in the PM phase at $200 \mathrm{~K}$, there is no critical scattering present as shown by the open circles. The magnetic correlations in the PM phase change to yield neutron scattering which peaks at the Bragg points for the SDW (refer to open circles in the lower panel). In the AFI phase, there are no magnetic correlations of the SDW type as shown by the solid circles at $130 \mathrm{~K}$. The PM-AFI transition, therefore, is accompanied by an abrupt switching between two different kinds of magnetic correlations $[17,12]$.

The paramagnetic spin fluctuations in the insulator (PI) also have different spatial correlations from those in the low temperature AFI phase, as shown in Fig. 5 for a Cr-doped $\mathrm{V}_{2} \mathrm{O}_{3}$ sample. Similar to those in the $\mathrm{PM}$, they peak in a region of q-space displaced from nuclear Bragg points along the $\mathbf{c}$ axis (refer to the (10l) scan in the figure). Similar switching of magnetic correlations occurs at the PI-AFI phase transition $[17,12]$. Thus, the magnetic transition from either the PM or the PI to the AFI is not a conventional spin order-disorder transition, rather it appears that the spin Hamiltonian is abruptly altered at the AFI transition.

There are other anomalies in the dynamic spin correlations of the PI. As is evident from the broader peak width of constant- $E$ scans in the PI as compared to the PM (refer to the (10l) scans in Fig. 4 and 5), the correlation length in the PI is even shorter than that for the PM where electron-hole damping exists. This short correlation length amounts only to a nearest neighbor distance and shows no discernible increase as the AFI transition is approached by cooling the sample [12]. Nevertheless, these extremely short-range dynamic magnetic correlations involve a substantial magnetic moment, $0.81 \mu_{B}$ per $\mathrm{V}$, below $18 \mathrm{meV}$ at $205 \mathrm{~K}$. Therefore, they are not due to weak magnetic interactions $\left(J \ll k_{B} T\right)$.

All these results can be consistently explained, as pointed out by Rice [18], by considering the doubly degenerate $d$-orbitals at the Fermi level for each electron on a vanadium site. It has been shown more than twenty years ago that the extra degrees of freedom from orbital degeneracy have a profound effect on the Hubbard physics [19,20]. Specifically, the spin and orbital degrees of freedom strongly influence each other. In the AFI phase of $\mathrm{V}_{2} \mathrm{O}_{3}$, spin order 
develops simultaneously with order in the orbital occupations at V sites [20]. In the PI phase, orbital occupations fluctuate [18], thereby inducing magnetic interactions with fluctuating signs. It is no wonder then that dynamic spin correlations in the PI are of large amplitude yet remain extremely short-ranged $[12,17]$. This is very different from the one-band case where insulating antiferromagnetism is described by a fixed Heisenberg spin Hamiltonian [21]. It would be interesting to study localized spin systems with fluctuating exchange interactions, and details of our experimental results in the PI remain to be understood [12].

In summary, the metal-insulator transition fundamentally alters the nature of antiferromagnetism in $\mathrm{V}_{2} \mathrm{O}_{3}$. Contrary to widely circulated beliefs, the magnetism is itinerant in nature and is still determined by the Fermi sea in the nearly localized Fermi liquid of metallic $\mathrm{V}_{2} \mathrm{O}_{3}$. The first order AFI transition is primarily an orbital ordering transition. Strong spin-orbital coupling results from different overlap integrals between anisotropic $d$-orbitals on neighboring sites.

We gratefully acknowledge discussions with T. M. Rice, S. K. Sinha, Q. M. Si, G. Sawatzky, A. Fujimori, L. F. Mattheiss, G. Kotliar, C. Castellani, M. Takigawa, R. J. Birgeneau, G. Shirane, S. M. Shapiro, J. Tranquada, C. Varma, A. J. Millis, J. W. Lynn, and R. W. Erwin. Work at BNL was supported by DOE under Contract No DE-AC0276CH00016, at JHU by the NSF through DMR-9453362, at ORNL by DOE under Contract No DE-AC05-84OR21400, at Purdue by MISCON Grant No DE-FG02-90ER45427. will be changed 


\section{REFERENCES}

[1] A. Schilling, M. Cantoni, J. D. Guo and H. R. Ott, Nature 363 (1993) 56; Z. J. Huang et al., Physica C 217, 1 (1993).

[2] S. Jin, H. M. O'Bryan, T. H. Tiefel, M. McCormack and W. W. Rhodes, Appl. Phys. Lett. 66, 382 (1995).

[3] J. Feinleib and W. Paul, Phys. Rev. 155, 841 (1967); M. Föex, Compt Rend. 223, 1126 (1946); F. J. Morin, Phys. Rev. Lett. 3, 34 (1959).

[4] N. F. Mott, Metal-Insulator Transitions, 2nd Ed. (Taylor \& Francis Ltd, London 1990).

[5] D. B. McWhan, T. M. Rice and J. P. Remeika, Phys. Rev. Lett. 23, 1384 (1969).

[6] D. B. McWhan, J. P. Remeika, T. M. Rice, W. F. Brinkman, J. P. Maita and A. Menth, Phys. Rev. Lett. 27, 941 (1971); D. B. McWhan, J. P. Remeika, J. P. Maita, H. Okinaka, K. Kosuge, and S. Kachi, Phys. Rev. B 7, 326 (1973); S. A. Carter, T. F. Rosenbaum, P. Metcalf, J. M. Honig and J. Spalek, ibid. 48, 16841 (1993).

[7] D. B. McWhan and T. M. Rice, Phys. Rev. Lett. 22, 887 (1969); D. B. McWhan, A. Menth, J. P. Remeika, W. F. Brinkman, and T. M. Rice, Phys. Rev. B 7, 1920 (1973); Y. Ueda, K. Kosuge and S. Kachi, J. Sol. Stat. Chem. 31, 171 (1980).

[8] Y. Ueda, K. Kosuge, S. Kachi, T. Shinjo and T. Takada, Mat. Res. Bull. 12, 87 (1977); J. Dumas and C. Schlenker, J. Magn. Magn. Mat. 7, 252 (1978).

[9] W. Bao, C. Broholm, S. A. Carter, T. F. Rosenbaum, G. Aeppli, S. F. Trevino, P. Metcalf, J. M. Honig and J. Spalek, Phys. Rev. Lett. 71, 766 (1993).

[10] W. Bao, C. Broholm, J. M. Honig, P. Metcalf and S. F. Trevino, Phys. Rev. B 54, R3726 (1996).

[11] E. Fawcett, Rev. Mod. Phys. 60, 209 (1988). 
[12] W. Bao, C. Broholm, G. Aeppli, S. A. Carter, P. Dai, T. F. Rosenbaum, J. M. Honig, P. Metcalf and S. F. Trevino, to be published (1997).

[13] A. J. Millis, Phys. Rev. B 48, 7183 (1993); S. Sachdev and J. Ye, Phys. Rev. Lett. 69, 2411 (1992).

[14] T. Moriya and K. Ueda, J. Phys. Soc. Jpn. 63, 1871 (1994); K. Nakayama and T. Moriya, ibid. 56, 2918 (1987); H. Hasegawa, ibid. 38, 107 (1975); H. Hasegawa and T. Moriya, ibid. 36, 1542 (1974).

[15] R. M. Moon, Phys. Rev. Lett. 25, 527 (1970).

[16] R. E. Word, S. A. Werner, W. B. Yelon, J. M. Honig and S. Shivashankar, Phys. Rev. B 23, 3533 (1981).

[17] W. Bao, C. Broholm, G. Aeppli, P. Dai, J. M. Honig and P. Metcalf, Phys. Rev. Lett. 78, 507 (1997).

[18] T. M. Rice, in Spectroscopy of Mott Insulators and Correlated Metals, eds. A. Fujimori and Y. Tokura, (Springer, 1995).

[19] K. I. Kugel and D. I. Khomskii, Sov. Phys.-JETP 37, 725 (1973); M. Cyrot and C. Lyon-Caen, J. Phys. (Paris) C 4, 183 (1976).

[20] C. Castellani, C. R. Natoli and J. Ranninger, Phys. Rev. B 18, 4945 (1978).

[21] P. W. Anderson, Solid State Phys. 14, 99 (1963). 


\section{FIGURES}

FIG. 1. The composition-temperature phase diagram of $\mathrm{V}_{2} \mathrm{O}_{3}$ [7]. The solid and dashed lines denote first and second order phase transitions respectively.

FIG. 2. (left) Magnetic structure in the SDW state of $\mathrm{V}_{2-y} \mathrm{O}_{3}$, from [9]. The incommensurate spiral has wave vector $(0,0,1.7)$ using the conventional hexagonal unit cell shown here. (right) Magnetic structure in the AFI state of $\mathrm{V}_{2} \mathrm{O}_{3}$, according to [15]. The magnetic wave vector is $(1 / 2,1 / 2,0)$.

FIG. 3. Intensity contour of $S(\mathbf{q}, \omega)$ along $(10 l)$ direction at $1.4 \mathrm{~K}$ for $\mathrm{V}_{1.973} \mathrm{O}_{3}$ [17]. The $\mathbf{q}$ range spans a whole Brillouin zone with nuclear Bragg points (10̄) and (104) at the ends. The two points at $(1,0, \overline{0.3})$ and $(1,0,2.3)$ are magnetic Bragg points. Intensity is indicated by the bar on the right side in units of $\mu_{B}^{2} / \mathrm{meV}$ per unit cell.

FIG. 4. Constant $\hbar \omega$ scan along $(1 / 2,1 / 2, l)$ (upper panel) and (10l) (lower panel) in the PM (open circles) and the AFI (solid circles) phases for $\mathrm{V}_{2} \mathrm{O}_{3}$, from [17].

FIG. 5. Constant $\hbar \omega$ scan along $(1 / 2,1 / 2, l)$ (upper panel) and $(10 l)$ (lower panel) in the PI (open circles) and the AFI (solid circles) phases for $\mathrm{V}_{1.94} \mathrm{Cr}_{0.06} \mathrm{O}_{3}$, from [17]. 


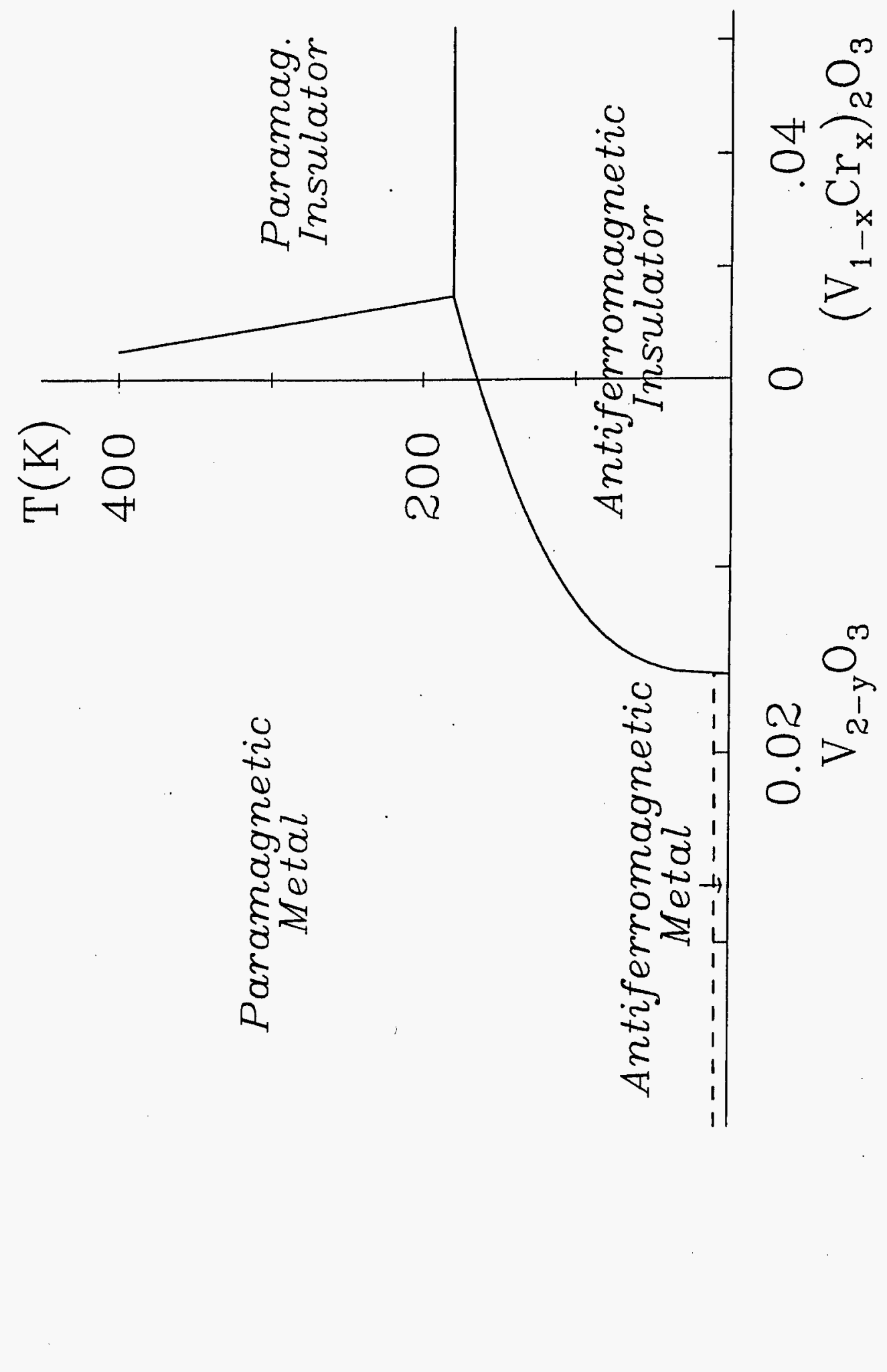



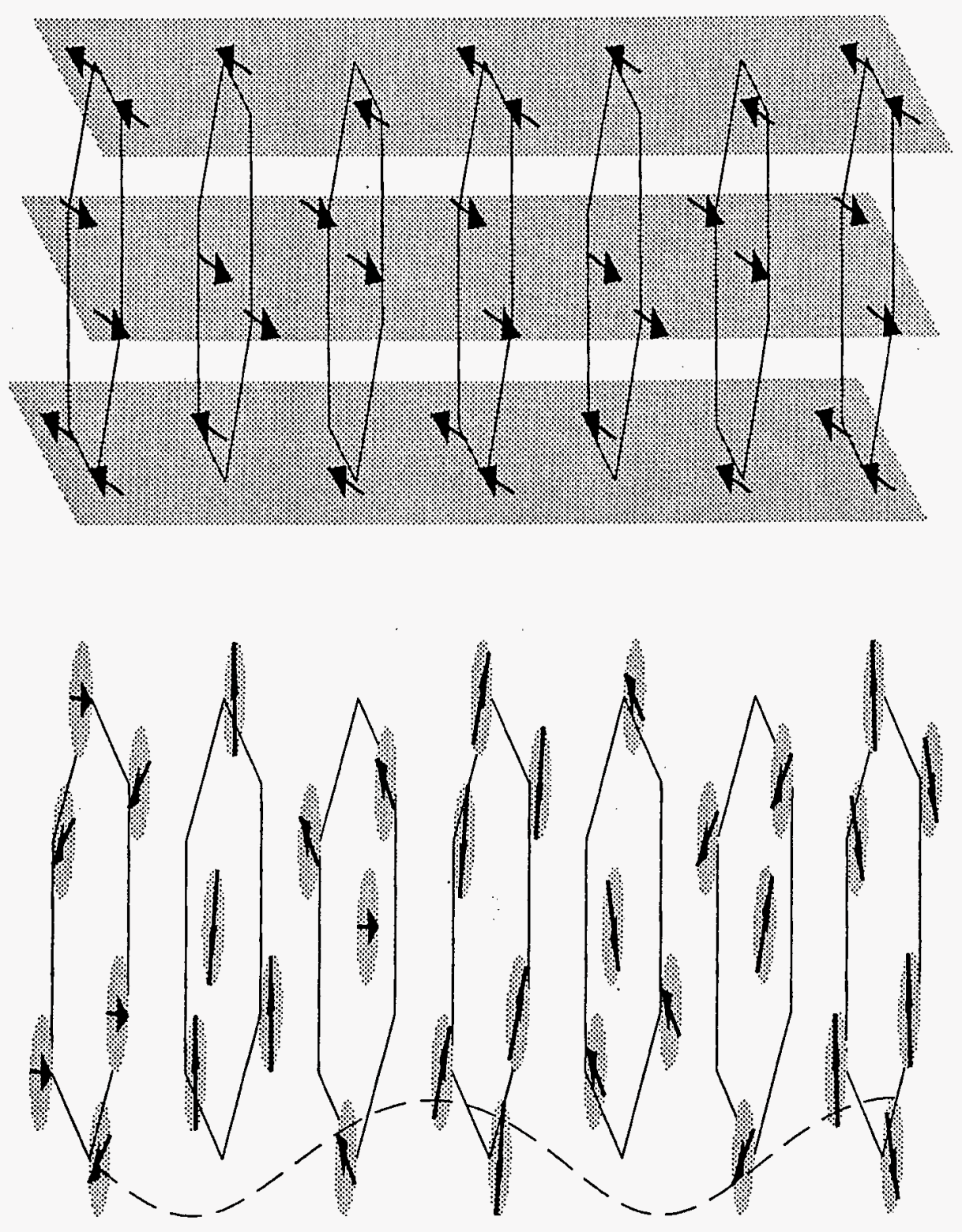

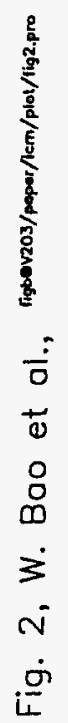



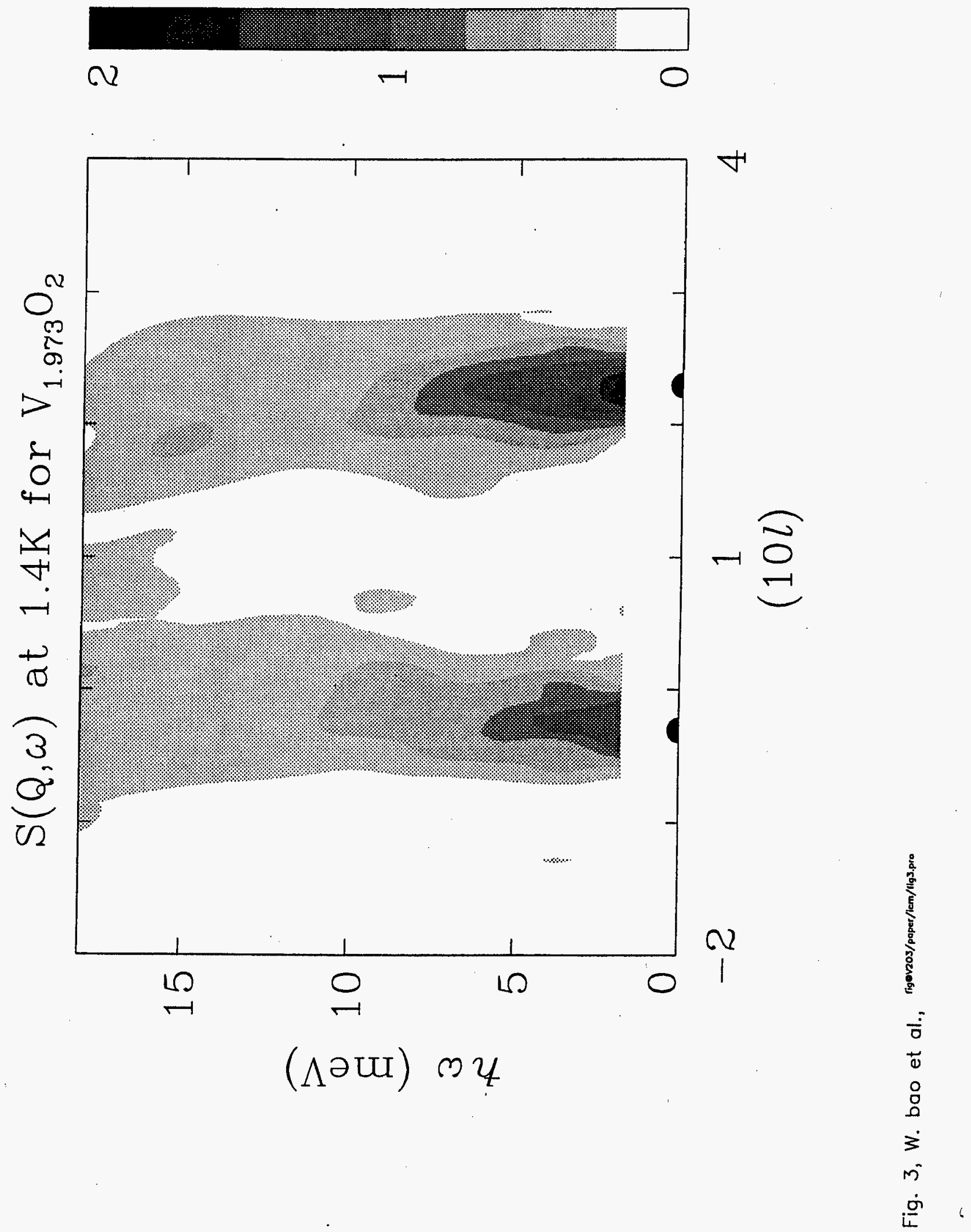


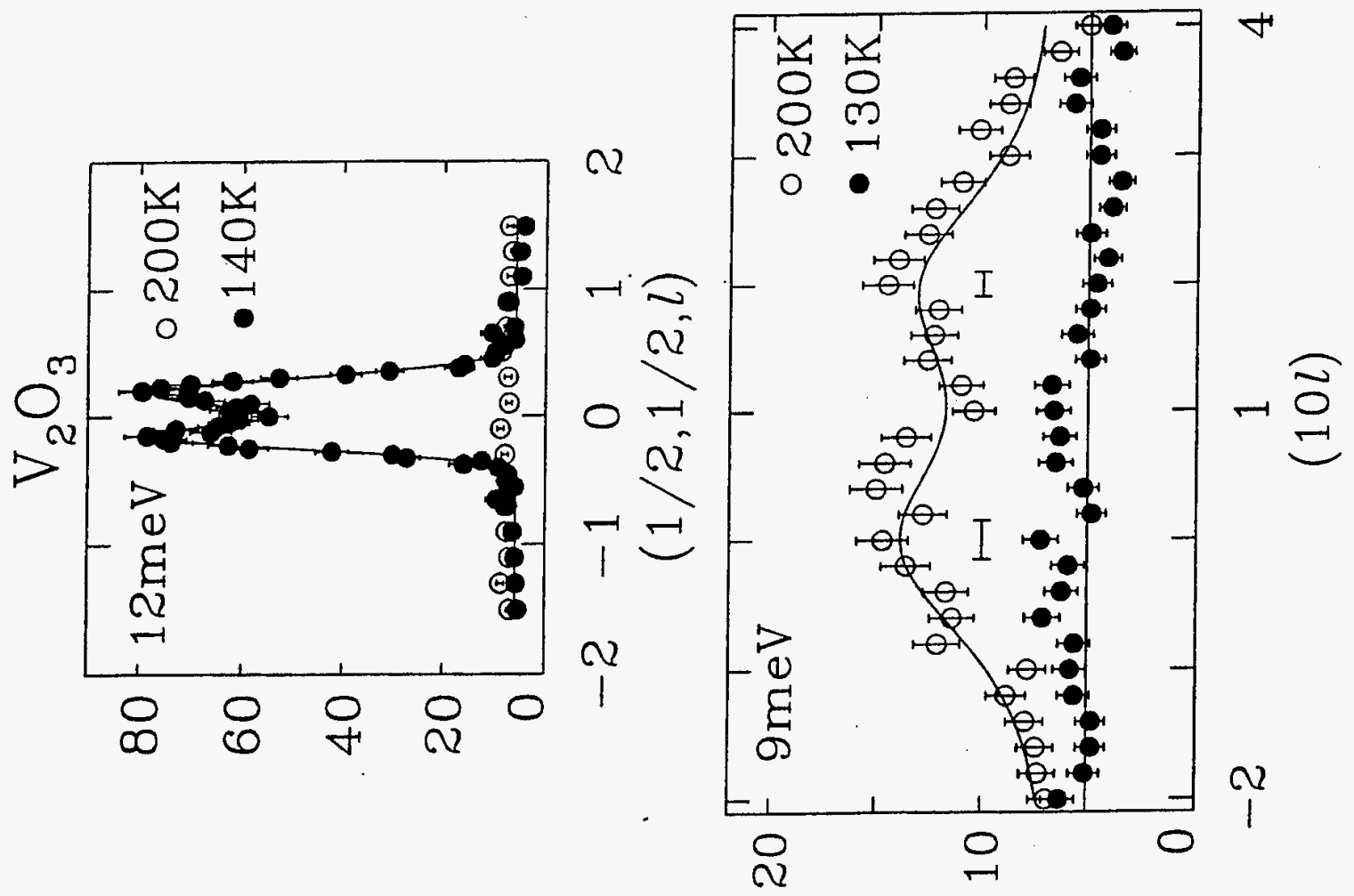

uṭu dəd słunoग 


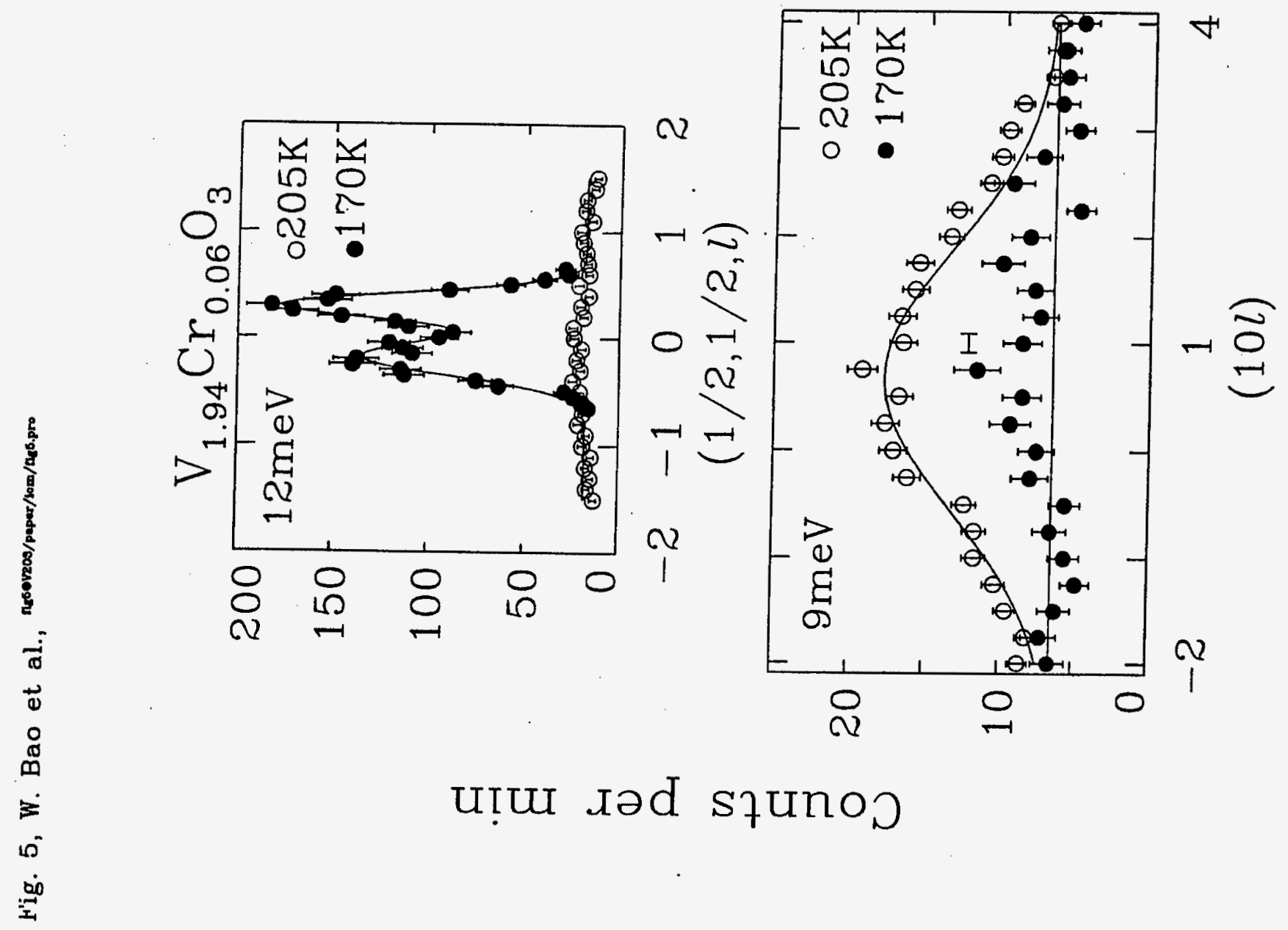




\section{M98004823 \\ ||||||||||||||||||||||||||||||||||||||||||||||||||||}

Report Number (14) ORNL/CP--97289

$\frac{\text { BNL- } 64816}{\text { CONF-970713 }}$

Publ. Date (11) Doe $\frac{199707}{E R}$

Sponsor Code (18) ER , XF

UC Category (19) UC-4O4, DOE/ER 